\title{
Modification of Oxide Thin Films with Low-Energy Ion Bombardment
}

\author{
Oscar Rodríguez de la Fuente
}

Additional information is available at the end of the chapter

http://dx.doi.org/10.5772/66651

\begin{abstract}
We review in this chapter the use of low-energy ion bombardment (LEIB) in oxide thin films. In most cases, radiation effects in oxides are ultimately based on the preferential sputtering of the oxygen anions, yielding a chemically reduced oxide. The physics governing the processes in the low ion energy range will be briefly commented here. Also, general uses and applications of LEIB are reviewed here, focusing later in those specific applications on oxide layers. LEIB in oxides has supported, for instance, the fabrication of conductive transparent layers on top of semiconductors or the formation of self-organized morphological surface patterns. Finally, we show a novel application of LEIB when applied on single-crystalline surfaces of some oxides, which is the formation of an epitaxial thin film of the corresponding suboxide. For instance, we show how ion bombardment transform the surface of $\mathrm{TiO}_{2}(110)$ into an epitaxial $\mathrm{TiO}(001)$ thin film.
\end{abstract}

Keywords: low-energy ion bombardment, oxide, thin film, defect, epitaxy

\section{Introduction}

Ion irradiation of solids has been a research topic for decades. In the $\mathrm{keV}$ range, the maximum penetration depth in the material does not exceed a few nanometers, and ions in this range of energies have traditionally assisted in the preparation and analysis of surfaces: it has served as a cleaning tool in vacuum conditions and, combined with surface analysis techniques, in compositional depth analysis or to assist in the growth of thin films. But low-energy ion bombardment (LEIB) has been also a tool to controllably modify surfaces or thin films. It has several advantages over other surface modification methods: (a) its low penetration depth, which allows the modification of a shallow surface layer or a thin film, (b) the high degree of control by choosing the type of ion, energy, flux, and dose received by the sample, and (c) the possibility of modifying very small surface areas by using masks or focused beams. 
We focus in this chapter in this low-energy range (from a few hundred $\mathrm{eV}$ to a few $\mathrm{keV}$ ), showing the capabilities of the technique for a specific family of systems: thin oxide films. Oxides are materials which display a huge plethora of physical and chemical properties, and in the form of thin films, they have been extensively studied in the last two decades. Its ample tolerance to defects favors the availability of compounds with different stoichiometries and properties. This is a source of complexity but also provides opportunities to explore many different properties in various fields, such as catalysis, magnetism, sensors, or electronics. LEIB stands as a very attractive experimental technique to modify the properties of oxide thin films by creating defects or inducing structural and compositional transitions.

Oxides are present in a large number of technological devices, and a growing number of applications will probably be found in the coming decades. For this to occur, a broad insight of their properties and a deep understanding of the role played by defects will be required. As in the case of silicon technology, where defect engineering is responsible for the precise and powerful control of electronic properties, defect engineering in oxides is for sure the tool to be exploited to enhance the performance of any present or potential device based in oxides. This is by no means a novel statement, for it has been well known for many decades that the presence of defects in oxides determines many of their properties. However, two challenges nowadays are (a) to acquire a more profound knowledge of defects properties in oxides and (b) to be able to introduce them in the surface of the material in a controlled way. LEIB is a good tool for this purpose but goes beyond the simple generation of defects. It also induces the formation of new structural phases or the generation of textured and nanostructured surfaces, as we will describe in this chapter.

\subsection{Historical remarks}

Ion sputtering, or the ejection of atoms from surfaces with energetic ions, is a phenomenon reported more than 150 years ago. The effect of sputtering was already described in 1853 by Grove [1], when he observed that the cathode metal located inside discharge tubes gradually coated the inner walls. The coating was caused by the positive ions of the discharges hitting the negatively charged metal cathode, sputtering atoms away from it subsequently coating the glass. However, this was not unambiguously verified until 1902, about 50 years later, when Goldstein [2] performed the correct experiments. In the meantime, other hypotheses were considered (such as that thermal evaporation induced by the discharge). But the effect of atomic collisions from positively charged ions was finally acknowledged. It was in the mid-1900s that a quantitative description of the sputtering process was reached and a sound theory was developed. Since then, hundreds of works have continued improving the existing theories and performing new experiments about surface sputtering. Some of these works are reviewed in the excellent work by Sigmund [3]. The purpose of many of these investigations has been the application of low-energy ion bombardment on a growing number of materials, where of course we can meet oxides.

\subsection{Organization of the chapter}

In this work, we start introducing the most important theoretical concepts related to ion bombardment, focusing around the low-energy range. As we are dealing with oxides, the 
concept of preferential sputtering of multicomponent materials is discussed. Then, we present some of the many applications or current uses of the technique for all types of materials, such as the modification of the surface topography or the controlled introduction of surface defects. Next, we invoke those uses reported in the literature about oxide surfaces and thin films, grouped in two big sets: (a) those examples exclusively describing morphological, structural, and chemical changes of the surfaces and (b) those works reporting other associated physicochemical properties, such as electrical conductivity. Finally, we present two applications recently carried out in our research group, which is the formation of singlecrystalline epitaxial thin films of a suboxide when the surface of the corresponding oxide is ion bombarded: we report the formation of a $\mathrm{TiO}(001) / \mathrm{TiO}_{2}(110)$ thin film and a $\mathrm{Fe}_{3} \mathrm{O}_{4}(111) / \alpha-$ $\mathrm{Fe}_{2} \mathrm{O}_{3}(0001)$ bilayer.

\section{Theoretical background}

We present in this section a few theoretical concepts about some of the most relevant physical processes taking place during the collision of ions with solid surfaces. For a more profound study of the physics involved, the reader is referred to other works in the literature $[3,4]$.

\subsection{Ion stopping}

Several mechanisms are active when an ion enters a solid material, dissipating its initial energy. Ion stopping in a solid can take place by interactions with both the electrons and ions of the crystal. The physical quantity describing the interaction of the ion with the solid is the stopping power or cross section $S(E)$, which depends on the ion energy, and so it can be divided into two terms, an electronic $(e)$ and a nuclear $(n)$ term:

$$
S(E)=S_{e}(E)+S_{n}(E)=-\frac{1}{N}\left(\left(\frac{d E}{d x}\right)_{e}+\left(\frac{d E}{d x}\right)_{n}\right)
$$

As Eq. (1) states, $S(E)$ can be also described in terms of the energy loss rate $d E / d x$, being $N$ the number density of atoms in the crystal. It is interesting to note that, as a universal reference, energy losses are typically of several hundreds of eV/nm in the low-energy range.

Collisions of the incident ion with electrons are more frequent, but in the range of low-tomedium energies, they have a little effect on the main trajectory of the ion (because of their small mass). In these ranges, electronic stopping has a comparable effect to that of a viscous flow continuously reducing the kinetic energy of the ion. In contrast, the interaction with the ions is more discrete, taking place between elastic consecutive collisions. For these nuclear losses, a simple elastic model using conservation of momentum describes the maximum transferred energy $T_{\max }$ from an incident atom to the struck atom as

$$
T_{\max }=\frac{4 E_{0} M_{1} M_{2}}{\left(M_{1}+M_{2}\right)^{2}}
$$

where $M_{1}$ and $M_{2}$ are the masses of both atoms and $E_{0}$ is the kinetic energy of the incident one. 
Depending on the nature of the collision, the effect produced has been typically classified in three regimes. In the single knock-on regime, the incident ion generates a small cascade of events, where atoms sequentially transfer their energy. Eventually, one of the atoms surpasses the lattice binding forces and is ejected out of the solid. But if the ion is sufficiently energetic, secondary cascades are generated, each of which can produce their own sputtered atoms. But the whole process can still be described as a sum of individual events. This is called the linear cascade regime. If, instead, the energy density of the collision is very high and most of the atoms located in a region are severely displaced from their equilibrium positions, looking more like a melted fluid, the regime is called thermal spike. The range where a given event is placed depends not only on the ion energy but also on the ion mass. As can be deduced from Eq. (2), a light ion $\left(\mathrm{H}^{+}, \mathrm{He}^{+}\right.$, etc. $)$is very inefficient in its energy transfer $T_{\max }$ and enters the spike regime at very high ion energies. Most of the cases considered in this chapter, with medium-mass ions (mostly $\mathrm{Ar}^{+}$) with up to a few $\mathrm{keV}$ of energy, lie in the single knock-on or linear cascade regime.

\subsubsection{Ion range}

To evaluate the spatial range of the area of the ion-bombarded material, the most important concept is that of the ion range $R$. The ion range in a solid is the integrated distance traveled by the ion before it stops after reducing its energy to zero, and so it is defined as

$$
R=\int_{E_{i}}^{0} \frac{d E}{d E / d x}
$$

Another useful magnitude is the projected range $R_{p^{\prime}}$ defined as the distance of $R$ projected along the incoming direction of the ion before entering the solid. In the case of normal incidence, $R_{p}$ is just the depth at which the ion remains implanted, which is not necessarily the distance traveled $R$. Figure 1 shows schematically these parameters.

\subsection{Partial sputtering yield}

The sputtering yield is the average number of atoms leaving the solid per incoming ion. An atom is sputtered off the solid when it receives an impact which overcomes the threshold energy and has the possibility to exit the solid and enter the vacuum region. While the former condition is achieved by many atoms involved in cascade collisions, the last condition is only fulfilled by a few atoms close to the surface with their linear momentum pointing away toward vacuum.

As we are dealing with oxides, the concept of preferential sputtering is the most important one. Ion irradiation of multicomponent surfaces always involves compositional changes. Many works in the literature deal with these types of processes, which we will briefly expose here (the reader is referred to Ref. [5] for an excellent review of the topic). The most important physical quantity in a multicomponent material is the partial sputtering yield $Y_{i}$ of element $i$, which is the average number of ejected $i$ atoms per incoming ion. Mathematically, it can be defined as

$$
Y_{i}=\int_{0}^{-\infty} p_{i}(z) N_{i}(z) d z
$$




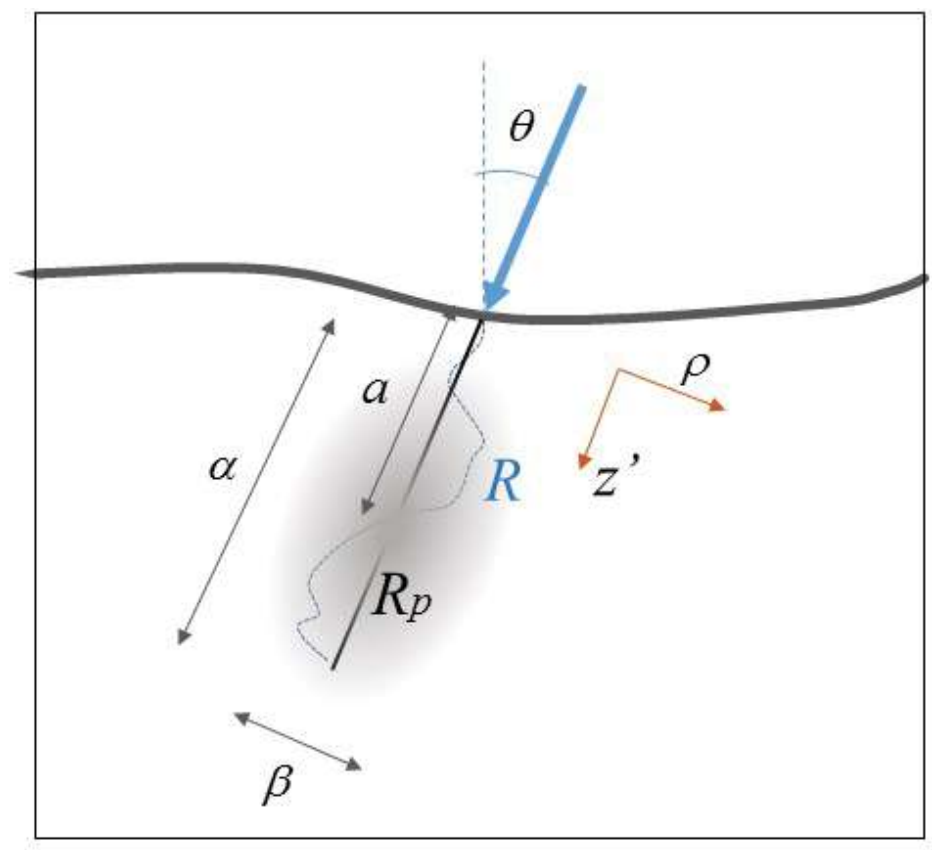

Figure 1. Scheme of the main parameters from Eqs. (3) to (6) for an ion impinging a surface at an angle $\theta$. The center of the ellipsoid represents the region where the ion deposits most of its energy.

where the partial sputtering yield depends on the density $N_{i}(z)$ of atoms of type $i$ (in atoms per unit volume) at a given depth $z$ below the surface and with a sputtering probability $p_{i}(z)$, which decays to zero with increasing depth.

\subsubsection{Preferential sputtering of oxygen}

In a simple binary oxide, the ratio between the partial sputtering yields can be described as Eq. (4)

$$
\frac{Y_{M}}{Y_{O}}=\frac{N_{M}}{N_{O}}\left(\frac{M_{O}}{M_{M}}\right)^{2 m}\left(\frac{U_{O}}{U_{M}}\right)^{1-2 m}
$$

where $N_{i}$ are the atomic densities, $M_{i}$ are the masses, and $U_{i}$ are the binding energies of the species ( $M$ stands for the metal cation and $O$ for the oxygen anion). $m$ is an exponent which characterizes the type of interatomic potential describing the collision and varies from 1 to 0 in the range from high to very low energies, respectively. Eq. (5) predicts that oxygen atom (the lightest atom and also frequently the most weakly bound to the crystal lattice) will be more easily ejected off the solid. However, Eq. (5) only describes a transient state. If the ion penetration depth is much smaller than the thickness of the sample bombarded, a steady state (after a sufficiently large ion fluence) will be reached where the bombarded material loses elements 
in a ratio equal to its bulk composition. That is a simple consequence of mass conservation, and it is achieved if mass diffusion to long distances is not relevant.

According to the effects previously addressed, a clear consequence of ion sputtering is the formation of a modified layer, not only in terms of its structure or its density of defects but also in terms of chemical composition. A fact to take into account is that the depth of the altered layer may be larger than the penetration depth of the incoming ions. The reason can rely on thermal or bombardment-induced diffusion. Dissipated energy, either from the ion beam or from mechanical agitation from collisions, enhances diffusion. The diffusion is driven by a chemical gradient, which can be present even from many atomic layers below $R_{p}$ (so, relatively far from the direct influence of the impinging ion).

\section{Applications of low-energy ion bombardment}

\subsection{Surface cleaning and sample preparation}

Probably, the most extended application of ion bombardment is the cleaning of the surfaces prior to their analysis or before another physical or chemical process. For instance, those surfaces of hard drilling tools which are to be coated with nitrides by means of physical vapor deposition (PVD) are usually exposed to $\mathrm{Ar}^{+}$prior to the coating. The reasons are the cleaning of the surface and the enhancement of atomic rugosity, which will favor mechanical adhesion of the coating, and thus will warrant a longer life of the tool. This process is one of the many examples in surface science and engineering which requires LEIB. Another extended use occurs for transmission electron microscopy (TEM), which requires thin (electron transparent) samples. The final stage is usually carried out with ion bombardment, usually named ion milling.

\subsection{Secondary ion mass spectroscopy (SIMS)}

Ion bombardment, sometimes in conjunction with surface analysis techniques, has been traditionally used to obtain surface composition. Secondary ion mass spectroscopy (SIMS) analyses, with the help of a mass spectrometer, the nature of the atoms sputtered from a surface with an ion beam [6]. It is a useful technique to carry out depth profiling studies, especially if combined with XPS or Auger spectroscopy. However, the change in the chemical state of the surface, induced by the ion beam, must always be taken into account (except if the surface layer or layers to be analyzed are single component).

\subsection{Improvement of thin-film growth}

The simultaneous combination of thin-film growth (by means of any vacuum technique) and LEIB has been frequently employed to explore different states of the film grown and to improve the required properties. The effect of the incoming energetic ions has a profound influence in the morphology of the film, as well as in its defect density, which in turn modifies 
many other properties. The technique, usually called ion-beam-assisted deposition (IBAD), can produce a number of beneficial changes in several characteristics of the film, such as density, texture, residual stresses, adhesion, or crystalline order. Of course, this technique has been applied to thin films of oxides [7]. In most cases, the main role of the ions of the beam is to deposit energy, creating defects and inducing diffusion and mobility. But in some other cases (this is usually named reactive IBAD), the second role of the atoms in the beam is to chemically react with surface species to form compounds.

\subsection{Controlled generation of surface defects}

If bulk defects control many properties of solids (dislocations in the case of mechanical properties of metals or point defects in the case of optical properties of many oxides, to mention two time-honored examples), surface defects may as well control some surface physicochemical properties. The most paradigmatic example is that of the so-called active centers [8], which control some catalytic conversions and which have been proposed to be surface steps [9] or vacancies. A profound knowledge of the physical and chemical properties of surface defects requires a well-known process to generate them controllably, and LEIB is a good tool for that purpose [10-12]. Under the correct parameters (energy, dose, temperature, etc.), it can generate a broad spectrum of surface defects (surface vacancies, adatoms, linear steps, vacancy islands, etc.), which can be subsequently studied for whatever phenomenon of interest [13, 14]. Also, the kinetics of flattening of an initially rough surface, with the roughness induced with LEIB, has been studied for different types of materials (among them, also oxides). For instance, for the case of $\mathrm{TiO}_{2}(110)$ and after low ion doses (only 0.5 monolayers were sputtered), a power scaling law has been found for the high-temperature evolution of the width $w$ of the surface terraces as $w \sim t^{0.24}[15]$.

\subsection{Surface nanopatterning}

One of the first phenomena observed on ion-bombarded surfaces was the formation, in some cases, of ordered structures with a well-defined periodicity of a few tens of nanometers [16]. These patterns, usually in the form of ripples, may develop spontaneously from an initially flat surface as a result of several competing mechanisms which are active in a wide variety of materials. This phenomenon soon caught the attention of the scientific community for its interest and potential applications. The method just requires an ion beam homogeneously irradiating a surface and can easily pattern macroscopic areas of the order of $\mathrm{mm}^{2} \mathrm{or} \mathrm{cm}^{2}$ (once the correct processing conditions have been achieved). Thus, the absence of masks, complicated nanolithographic methods, or focused ion beams is very attractive from the technological point of view.

As already mentioned, the self-organized nanostructures spontaneously emerge under those experimental conditions which promote a clearly balanced competition between opposed kinetic processes occurring at the surface. Ion bombardment induces surface disordering and roughening, while surface diffusion favors smoothing and defect annihilation. These two main opposed trends, acting under nonequilibrium conditions and summed with other 
mechanisms or condition characteristic of each type of surface, determine the evolution and the final morphology of the surface.

The family of theories describing surface patterning is too ample to be mentioned here, but we can briefly comment here on the most acknowledged model, which accounts for the formation of ripples under an off-normal incident ion beam. This is the Bradley-Harper (BH) model [17], which successfully explains the dynamics of ripple formation in isotropic and amorphous surfaces: the ripples are parallel to the incident direction of the beam under high incidence angles and perpendicular to the beam direction under angles close to be grazing. To understand the $\mathrm{BH}$ model, one must consider the distribution of surface defects created by ion collisions. This is described by the Sigmund model [18], which accounts, under a continuum model approach, for an ellipsoidal average distribution of energy deposited under ion impacts formulated as

$$
E\left(z^{\prime}, \rho\right)=\frac{\varepsilon}{(2 \pi)^{1.5} \alpha \beta^{2}} \exp \left(-\frac{\left(z^{\prime}-a\right)^{2}}{2 \alpha^{2}}-\frac{\rho^{2}}{2 \beta^{2}}\right)
$$

Under cylindrical coordinates, $z^{\prime}$ and $\rho$ are the radial and longitudinal components, oriented along the initial incidence ion direction. $\alpha$ and $\beta$ are the longitudinal and lateral spreadings of the spatial distribution, $a$ is the center of the ellipsoidal distribution, and $\varepsilon$ represents the total energy deposited. Figure 1 graphically displays these parameters. That part of the energy deposited close to the surface will create a surface vacancy by sputtering an atom off the solid. Bradley and Harper used the Sigmund distribution to account for the creation of surface defects, describing their effect on surface topography $h(x, y, t)$ with a partial differential rate equation, where $x$ represents the direction parallel to the projection of the ion beam on the surface:

$$
\frac{\partial h(x, y, t)}{\partial t}=-Y_{0}(\theta)+\frac{\partial Y_{0}}{\partial \theta} \frac{\partial h}{\partial x}+v_{\|} \frac{\partial^{2} h}{\partial x^{2}}+v_{\perp} \frac{\partial^{2} h}{\partial y^{2}}-K\left(\nabla^{2}\right)^{2} h
$$

The first term in Eq. (7) is the erosion rate $Y_{0}$ for a flat surface, whose explicit dependency with the incidence angle $\theta$ is considered in the second term. The third and fourth terms take into account the contribution to roughening with the most important mechanism: the curvature-dependent sputtering yield. The concave regions of the surface have a larger probability to be even more eroded by the ion beam. Surface atoms at the valleys are more easily sputtered than those at the crests, so that once a valley is initiated, its height difference with the crests continues growing. This is, very naively described, the surface instability necessary to start the formation of ripples. The last term considers surface smoothing by atomic self-diffusion.

The $\mathrm{BH}$ model is relatively simple, and more advanced equations, including higher order terms or surface anisotropy, have been developed in the last two decades. But the BH model, as described by Eq. (7), agrees well with many experimental results, including the rotation of the ripples depending on the incidence angle of the ion beam. The $\mathrm{BH}$ theory only considers amorphous and isotropic solids and ignores the existence of crystallographic directions and surface steps. Surprisingly, the BH theory has proven successful in many crystalline surfaces 
and has been a good starting point in other cases. However, surface anisotropy induces new effects and mechanisms to be taken into account. For instance, surface steps may limit diffusion via Ehrlich-Schwöbel barriers, or may determine etching rates at low grazing angles, as we will see later. In any case, ion beam patterning has been observed in different types of materials, including oxides. We will refer to those works on surface nanopatterning of oxides in a specific section later.

\section{LEIB of oxide surfaces and thin films}

Oxides are a colossal family of materials, both in the sense of the existing diversities and of the properties displayed. The specific and complex nature of the bond between the oxygen and the metallic cation favors the existence of a vast set of functionalities in oxides. Complicated interactions are present, which cross-link the different properties of the oxide. Also, there is a huge variety of defects in oxides, which are rather abundant, on the other hand. This circumstance enormously obscures the profound understanding of the physical mechanisms involved in oxide properties but also assists in the modification or control of these properties.

Oxide thin films lie at the core of many technological devices [19]. Indium-tin-oxide (ITO) conductive and transparent coatings are a good example of this statement. The modification of the surface morphology and the controlled introduction of defects in thin oxide films are tools to improve their response in their respective applications. LEIB can assist in this task.

\subsection{Modification of the morphology}

Oxides have been also used to spontaneously generate ordered nanostructures in their surfaces with LEIB. Indeed, the first reported example took place for a glass surface bombarded with $\mathrm{Ar}^{+}$at $4 \mathrm{keV}$ by Navez in 1956 [16]. The authors discovered at that moment the formation of ripples separated by tens of nanometers. For incidence angles close to normal incidence and up to $\theta=80^{\circ}$, the direction of the ripples was perpendicular to the incidence angle. For grazing angles, in contrast, the direction of the ripples was parallel to the ion beam. This was probably the first time that the ripple rotation mechanism, later explained in the $\mathrm{BH}$ theory, was observed. Recent works on amorphous $\mathrm{SiO}_{2}$ have found that, while the quantitatively results are similar [20], there are differences depending on the type of silica (fused silica, amorphized silica, or thermally grown $\mathrm{SiO}_{2}$ ). The wavelength dependence on the energy and the wavelength coarsening vary with the substrate, which in turn depends on their surface energies (see Figure 2). It is interesting to note that, for low incidence angles, the surfaces remain smooth.

Although we focus in this chapter in the use of low-energy ions, medium energies have been used to nanostructure oxide surfaces too, and we would like to briefly mention it. However, in those cases where the energy is of the order of $10-100 \mathrm{keV}$, new mechanisms arise in the 

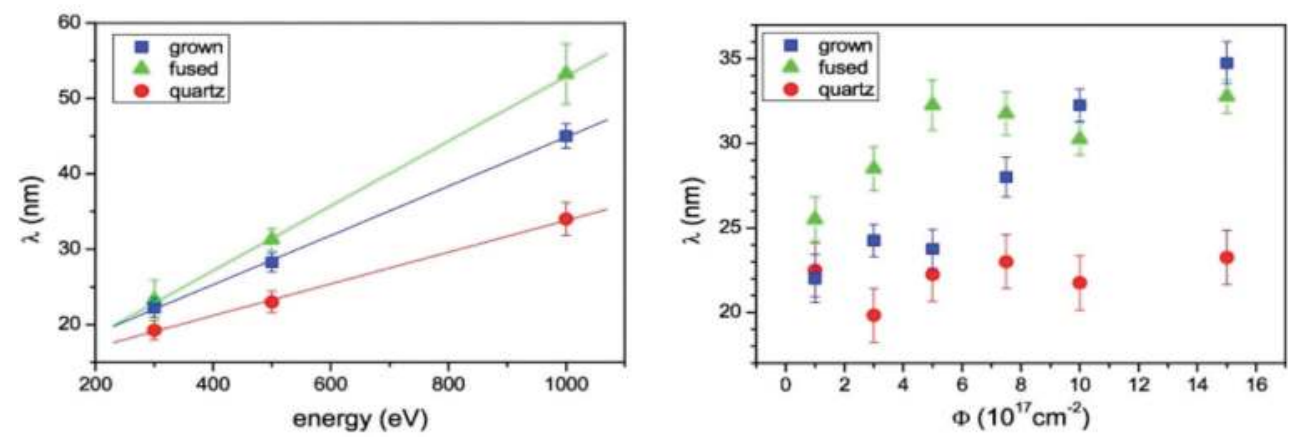

Figure 2. Ripple wavelength dependence on ion energy (left) and total ion dose (right) for three different $\mathrm{SiO}_{2}$ surfaces. The total dose for the graph on the left is $\Phi=1 \times 10^{18} \mathrm{~cm}^{-2}$. The incidence angle for both graphs is $45^{\circ}$. Reproduced with permission from Ref. [20].

evolution of the surface morphology, which are far from the mechanisms invoked by $\mathrm{BH}$ theories and similar. That is the case of anatase $\mathrm{TiO}_{2}$, for example, where different types of ions have been used to bombard the samples at different temperatures [21]. At these energies, the ion projected range $R p$ lies deep below the surface (of the order of tens of nanometers), and the agglomeration of point defects evolves into small voids in the first stages of ion bombardment. Further bombardment and void growth transform the initially flat surface into a morphology of void and mounds or nanorods aligned with the ion beam. In contrast, for the case of low-energy ions, the evolution of morphology does not include void formation, but is more limited to the formation and evolution of defects in the upper layers of the material.

Since $\mathrm{TiO}_{2}(110)$ is the most studied oxide surface, it can be understood that the nanopatterning of this surface has been addressed several times. In the case of low-energy ions, the morphology of the surface is, in many cases, rough and disordered if the bombardment takes place at room temperature. Diffusion is more limited in oxides than in metals, and there is no active mechanism to induce self-ordering at low $\mathrm{T}$. In the case of $\mathrm{TiO}_{2}(110)$ bombarded with $\mathrm{Ar}^{+}$with an energy between 0.5 and $1.5 \mathrm{eV}$ at room temperature, a disordered set of mounds is distributed across the surface [22]. These mounds are proposed to be chemically reduced oxide $\mathrm{Ti}_{2} \mathrm{O}_{3}$ regions, which align themselves along a given crystallographic direction under a thermal treatment between $700 \mathrm{~K}$ and $900 \mathrm{~K}$.

However, at higher temperatures, the formation of ordered ripples in $\mathrm{TiO}_{2}(110)$ at grazing incidence has been reported in Ref. [23]. The $\mathrm{TiO}_{2}(110)$ orientation has two nonequivalent in-plane surface directions: (001) and (1-10). At low incidence angles, the existence of surface steps is determinant in ripple formation. Indeed, sputtering from terraces is about one order of magnitude smaller than from step edges. Also, the sputtering yield or erosion rate of the different steps differs, and the consequence is that the total sputtering yield is larger when the beam is directed along the [1-10] direction than when it is oriented along the [001] direction. Despite all, at sufficiently long bombardment times, the beam direction does not affect too 
much the morphology of the formed ripples, somehow demonstrating the universality of the method for patterning large areas in different materials.

Another phenomenon which also recalls universality is the temperature-dependent reorientation of the ripples observed in $\mathrm{TiO}_{2}(110)$ [24], in the same way as for many metal surfaces. While at low $(150 \mathrm{~K})$ and high $(620$ and $720 \mathrm{~K})$ temperatures ripples are aligned along the beam direction, at $300 \mathrm{~K}$ they are aligned perpendicularly, as illustrated in Figure 3 . The same group has used LEIB as a tool to prepare $\mathrm{TiO}_{2}(110)$ surfaces with steps aligned along special orientations [25]. The method can be viewed as the equivalent to surface texturing with ion beams when the grains with higher sputtering yields destabilize. In this case, the ion beam under a low incidence angle preferentially erodes some specific steps, creating [1-10] steps. These are thermodynamically unstable but kinetically stabilized within this approach.
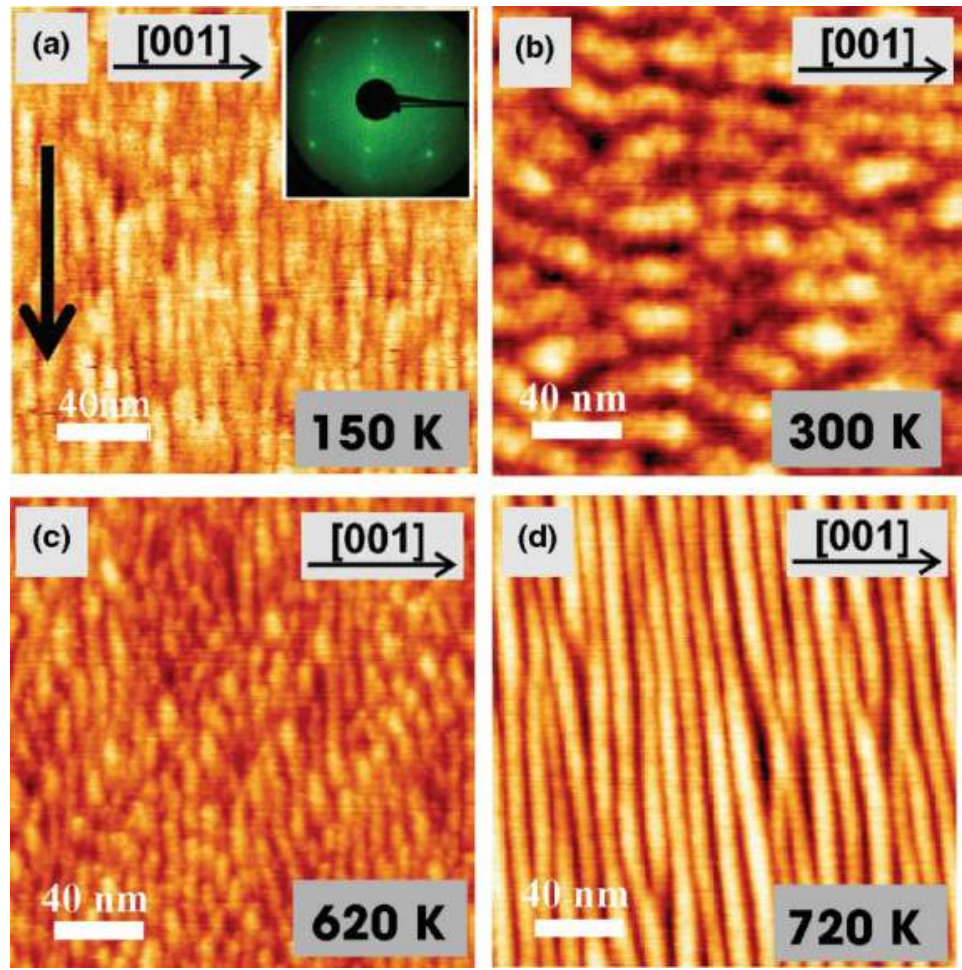

Figure 3. (a), (b), (c) and (d): STM images $\left(200 \times 200 \mathrm{~nm}^{2}\right)$ of $\mathrm{Ar}^{+}$-modified $\mathrm{TiO}_{2}(110)$ surfaces, where the bombardment has been carried out at different temperatures under an incidence angle of $75^{\circ}$, an ion energy of $2 \mathrm{keV}$, and a total dose of $9 \times 10^{16}$ ions $/ \mathrm{cm}^{2}$. The LEED pattern in (a) shows the crystalline order of the surface even after ion bombardment. All ripples are elongated along the beam projection, except for the case where bombardment has been done at RT. The black vertical arrow in (a) marks the projection of the beam on the surface and the horizontal black arrows mark the indicated crystallographic direction. Reproduced with permission from Ref. [24]. 
This work illustrates how grazing LEIB can be used to tune and texture certain surface steps, which can be relevant for applications sensitive to specific step orientations.

\subsection{Modification of the electronic properties}

Oxygen vacancy creation has been traditionally used to modify electronic properties of oxides. It is indeed a route to doping, since excess electrons (due to oxygen vacancies) can be transferred to the conduction band, transforming the material into a better conductor. This type of approaches has been sometimes referred to as vacancy engineering. The control to achieve the desired vacancy concentration can be gained through post-growth thermal annealings or during the growth, controlling experimental parameters such as the oxygen partial pressure or the laser energy if the material is grown by sputtering or by pulsed laser epitaxy (PLE) [26]. As already discussed, LEIB can be also helpful to control oxygen vacancies. Indeed, many of the most recent applications of LEIB on oxide surfaces are related to the modification of electronic and transport properties for different applications. In turn, other fundamental properties, such as the optical response, are modified as well. Most of the studies about oxides modified with LEIB have been accomplished on $\mathrm{SrTiO}_{3}$ and $\mathrm{TiO}_{2}$.

It is easy to understand that one of the oxides where LEIB has been most often employed to exploit its properties is $\mathrm{SrTiO}_{3}(\mathrm{STO})$, a transparent insulating perovskite with many intriguing characteristics and also frequently used as a substrate to grow other materials on it. A very interesting phenomenon is that LEIB can generate a conductive layer at its surface, and that has been the subject of several studies. There is a clear correlation between conductivity and the concentration of oxygen vacancies created by ion bombardment in STO [27]. The resistivity values of a modified nanometric layer obtained after bombarding a $\mathrm{SrTiO}_{3}(100)$ surface can follow a $\sim T^{2.5}$ law, dominated by the mobility of the carriers, and attain very low values (between $2 \times 10^{-4}$ and $6 \times 10^{-4} \Omega \mathrm{cm}$ at room temperature) without losing transparency [28]. In order to compare, some of the best conducting STO samples were previously produced by boron implantation at $100 \mathrm{keV}$, resulting in relatively thick and nontransparent conducting layers with resistivity values of about $0.01 \Omega \mathrm{cm}$ [29]. Thus, LEIB on STO is able to produce transparent conductive layers competitive with ITO layers, with an even smaller thickness.

This type of conductive layers formed by LEIB has also been reported in rutile $\mathrm{TiO}_{2}$ [30], reporting again high carrier densities and mobilities. The authors account for a high crystallographic order in the ion-bombarded region of the $\mathrm{TiO}_{2}(100)$ surface, which is not compatible with the rutile structure and which is attributed to the ordering of the vacancies produced by ion bombardment.

With respect to optical properties, hole levels in self-trapped states localized in the gap can be stabilized by conduction carriers (generated by oxygen deficiency) in Ar+bombarded single-crystalline STO [31]. Their recombination generates the emission of blue light at room temperature, and the emitting regions can be conveniently patterned. Cross-sectional TEM images show the formation of amorphous and modified (oxygen deficient) layers under the action of the ion beam (Figure 4). 


\section{Carbon coat}

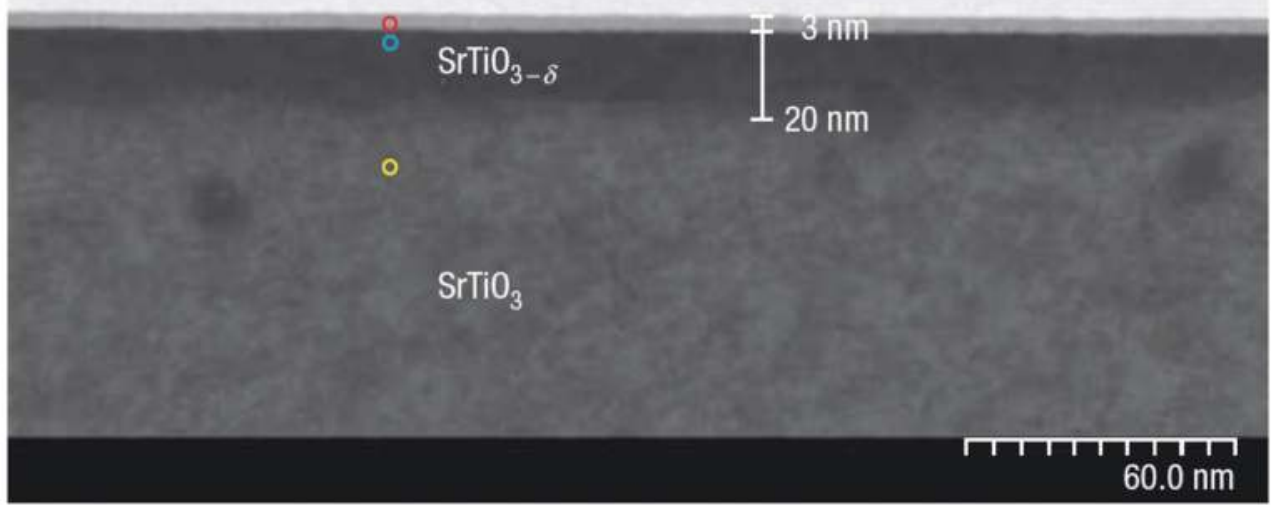

b

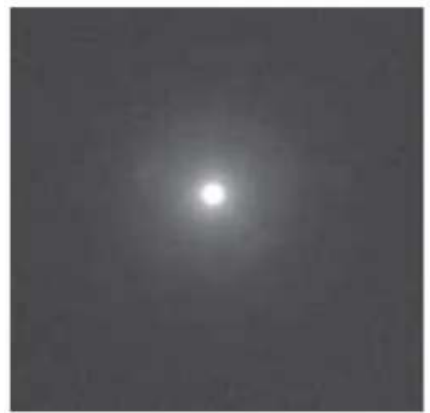

c

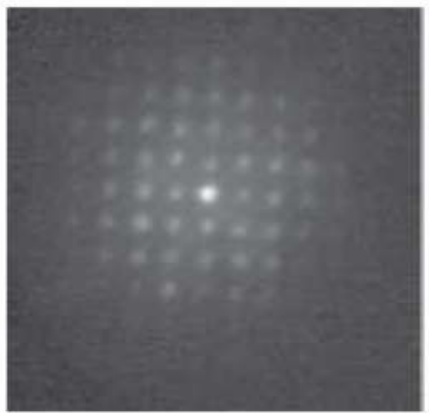

d

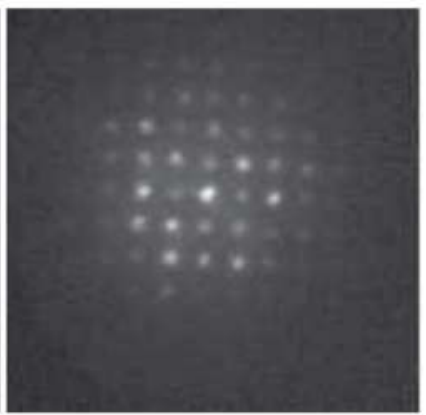

Figure 4. (a) TEM cross-sectional image from an $\mathrm{Ar}^{+}$-modified STO single crystal. (b)-(d) electron diffraction images of the (b) red, (c) blue, and (c) yellow regions of the material. Diffraction patterns from (c) to (d) are qualitatively similar, but the contrast in the real space image suggests that a modification indeed exists: the ion beam transforms the upper layer of pristine STO into an amorphous film (red region) and induces the formation of an oxygen-deficient $\mathrm{SrTiO}_{3-x}$ region with a thickness around 15-20 nm (blue region). Reproduced with permission from Ref. [31].

Under certain conditions, LEIB can also modify the optical properties of $\mathrm{TiO}_{2}(110)$ substrates, greatly enhancing its absorbance in the visible range and its luminescence [32]. The formation of self-organized crystalline nanodots at the surface and the generation of Ti interstitials justify this optical response. In general, this type of investigations opens a path to explore the field of oxide optoelectronics.

Another potential application where LEIB on oxides has found a niche is the generation of substrates suitable for resistive switching (RS) processes, which lie at the core of the nonvolatile 
data-storage memristor technology. This technology, which is still in its exploratory stages, is based in the existence of on/off states which depend on the electrical resistance level of the bit (rather than on the electrostatic or magnetic state). The state can be switched with external electric fields in oxides, which force a metal-insulator transition (MIT). $\mathrm{TiO}_{2}$ is a good candidate for this purpose, but it has been found that a previous forming step involving LEIB radically improves the RS process [33]. The conductive 2D layer generated consists in a set of self-organized grains with a locally reduced chemical composition. This distribution of grains is a kind of template where bipolar switching between a semiconductor and a metallic state (induced with a conductive atomic force microscopy (AFM) tip) is localized at the grains. Figure 5 illustrates a progressive chemical reduction and a declining electrical resistance of the $\mathrm{TiO}_{2}$ surface during ion bombardment. The same type of mechanism has been found in STO substrates [34] with rather low-energy $\mathrm{Ar}^{+}$ions (66-200 eV). LEIB greatly improves the resistive memory effect, if compared with pristine STO. In these cases, thermal annealings would create vacancies and would also improve the electrical conductance for RS processes. But the influence of LEIB is limited to the upper layers of the material and can be thus combined with focused ion beam methods or shadow masking to pattern surfaces.
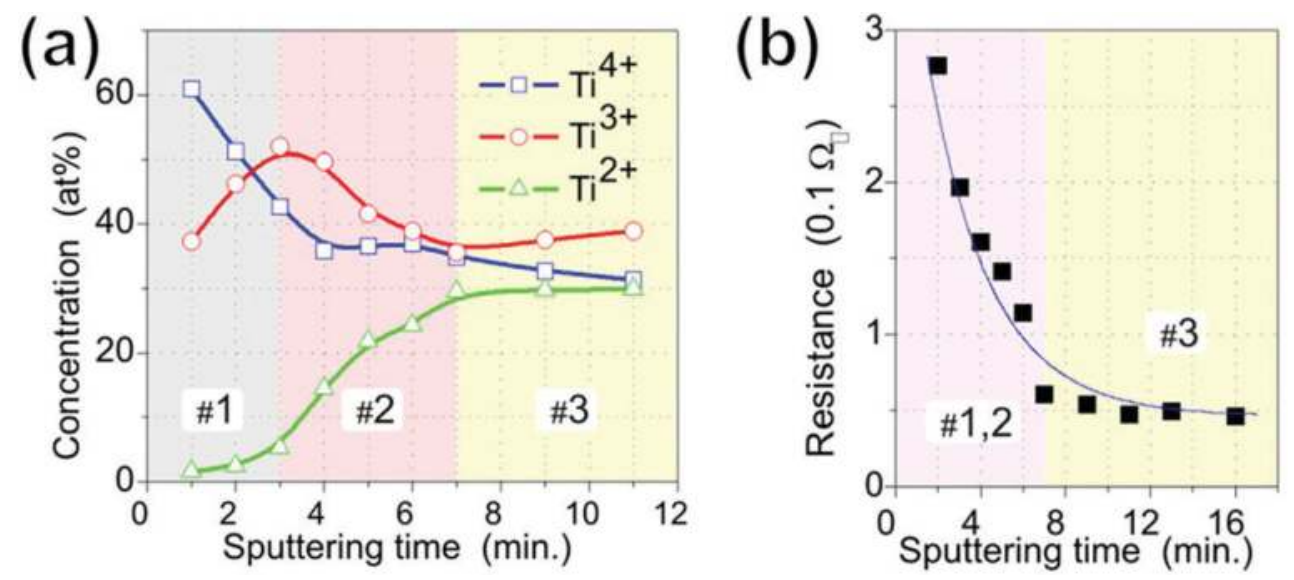

Figure 5. (a) Quantitative changes in the concentration of Ti cations with the sputtering time, as measured with XPS. (b) Behavior of the resistance (in $\Omega / \mu \mathrm{m}^{2}$ ) of the modified layer with sputtering time. Adapted with permission from Ref. [33].

Oxides exhibit outstanding magnetic properties, and in this respect, LEIB can be employed to modify the microstructure and consequently the magnetic response of the oxide layer. Bilayers of $\mathrm{Ni}_{80} \mathrm{Fe}_{20} / \alpha-\mathrm{Fe}_{2} \mathrm{O}_{3}$ grown on $\mathrm{SiO}_{2}$ exhibit, for instance, a different coercivity depending on the growth mode [35]. If ion bombardment is carried out on the $\alpha-\mathrm{Fe}_{2} \mathrm{O}_{3}$ layer before the growth of the $\mathrm{Ni}_{80} \mathrm{Fe}_{20}$ film, the uniaxial anisotropy of this layer is reduced, exhibiting a lower coercivity. This is a clear example of how the coupling between a ferromagnetic layer and an antiferromagnetic layer can be tuned with LEIB.

Regarding the modification of interfaces, we can mention that STO, in combination with other insulating oxides such as $\mathrm{LaAlO}_{3}(\mathrm{LAO})$, forms a heterostructure with remarkable conductive, 
magnetic, and superconductive properties at the interface. The surprising origin is the stabilization of an emerging 2D electron gas (2DEG). There are examples of the modification of the 2DEG in LAO/STO interfaces [36]. They have shown that the conducting state at the interface can be completely suppressed with $\mathrm{Ar}^{+}$bombardment at $150 \mathrm{eV}$. The conductivity can be then restored with a high-temperature annealing in the presence of oxygen. The authors suggest that the strain induced by Ar implantation causes local destabilization and partial amorphization. These disordered regions localize defect states where charges are trapped, in the same way as in other types of semiconductors. The annealing eliminates the Ar and heals defects, restoring the initial conductivity. The LEIB approach has been also used to create a 2D electron gas in an $\mathrm{Ar}^{+}$-bombarded $\mathrm{SrTiO}_{3}(100)$ surface [37], which shows an increase of the low-temperature magnetoresistance when the magnetic field $H$ points away from the surface. This and other effects are closely related to electron confinement, generated in this latter case by the modifications induced by ion bombardment.

\section{Formation of epitaxial thin films of oxides}

We present, in this final section of the chapter, a rather specific but quite surprising capability of ion bombardment, which we have successfully proven in a rutile $\mathrm{TiO}_{2}(110)$ substrate. As we have seen in previous sections, LEIB is a commonly used process to modify the physicochemical properties of oxide surfaces. In this respect, the literature has usually described the resulting material as a defective and chemically reduced version of the oxide, lacking a profound knowledge of the real structure. As all properties ultimately depend on the structure, the situation is highly undesirable. We show here a case where the structure of the abovementioned ion-bombarded surface is well defined after the modification.

The purpose of this section is to show that the use of LEIB on a surface of a single-crystalline oxide can induce the formation of a single-crystalline and epitaxial thin layer of the corresponding suboxide. For the case of $\mathrm{TiO}_{2}(110)$, we show here that high doses of $\mathrm{Ar}^{+}$bombardment of the single crystal produces a $10 \mathrm{~nm}$ thick film of $\mathrm{TiO}(001)$ [38].

It is well known that the progressive depletion of oxygen in polycrystalline materials during ion bombardment can, in some cases, induce the formation of a new phase with the cation in a lower oxidation state. In other words, the formation of a crystalline suboxide (i.e., with well-defined Bragg reflections) can be promoted. The main message of this section is that if the starting point is a single-crystalline surface (instead of ion bombarding a polycrystalline material) and if there is some kind of structural affinity or matching with a particular crystallographic orientation of the suboxide, this suboxide can nucleate and grow epitaxially coupled to the original oxide, being a thin epitaxial film the outcome of the process.

\subsection{Formation of a $\mathrm{TiO}(001)$ thin film on $\mathrm{TiO}_{2}(110)$}

Titanium dioxide is, with its different structures, a wideband semiconductor with many applications in technology. It has been also, along the years, a benchmark for the fundamental study of oxide surfaces. In fact, as we mentioned before, $\mathrm{TiO}_{2}(110)$ is the most studied oxide surface 
[39]. Regarding its optical properties, it is very transparent and has a high refractive index, which together with its stability, non-toxicity and the easy synthesis of small particles, justifies its extensive use as a white pigment. It also displays a very interesting photocatalytic activity. This property, along with its particular interaction with water, has fostered the investigation and the use of $\mathrm{TiO}_{2}$ as a self-cleaning coating [40]. As we have previously discussed, also in the field of resistive switching, the investigation with $\mathrm{TiO}_{2}$ has found a satisfactory feedback.

The starting point of the modification we describe here is a rutile $\mathrm{TiO}_{2}(110)$ clean and flat surface, which is ion bombarded with $\mathrm{Ar}^{+}$at $3 \mathrm{keV}$ at room temperature with doses up to $8 \times 10^{16}$ ions $\mathrm{cm}^{-2}$. Auger electron spectroscopy (AES) shows a clear chemical reduction: a decrease of the $\mathrm{O} / \mathrm{Ti}$ ratio and a shift of the $\mathrm{Ti}_{\mathrm{LMM}}$ and $\mathrm{Ti}_{\mathrm{LMV}}$ transitions to higher kinetic energies. The incidence of the ion beam is normal, and so the modified surfaces show a rough topography, but with no recognizable pattern or symmetry as seen with atomic force microscopy (AFM). However, X-ray diffraction of the bombarded surfaces already shows the emergence of a new reflection (not present in the pristine sample) compatible with the cubic rock salt titanium monoxide phase with its (001) crystallographic direction oriented along the surface normal (Figure 6a). Low-energy electron diffraction (LEED) measurements performed before and after the modification show the transformation of the rectangular surface diffraction pattern corresponding to $\mathrm{TiO}_{2}(110)$ to a different diffuse LEED pattern displaying square symmetry, with its main directions rotated $45^{\circ}$ with respect to the $\mathrm{TiO}_{2}(110)$ directions (Figure $6 \mathbf{b}-\mathbf{d}$ ).

Grazing incidence XRD (GIXRD) measurements help determine the crystalline orientations of both the dioxide and monoxide phases. HK scans in reciprocal space (those which explore the in-plane directions) show all the allowed reflections (not subject to extinction rules) of $\mathrm{TiO}(001)$ and $\mathrm{TiO}_{2}(110)$, confirming the $45^{\circ}$ rotation of the square surface lattice of $\mathrm{TiO}(001)$ with respect to the rectangular surface lattice of $\mathrm{TiO}_{2}(110)$ (blue and black lattices, respectively, of Figure 6e). L scans (to explore the out-of-plane direction) determine that the periodicity of the $\mathrm{TiO}$ phase is indeed the corresponding one. These observations confirm the ion-induced transformation of the upper layers of $\mathrm{TiO}_{2}(110)$ into $\mathrm{TiO}(001)$, with the particularity that both lattices are in registry. Being both lattices rotated $45^{\circ}$, the matching is almost perfect along the [001] direction of $\mathrm{TiO}_{2}(110)$. Along the [1-10] the mismatch is large (about 10\%), which clarifies the observations carried out with LEED, XRD, and scanning transmission electronic microscopy (STEM), all of them revealing the tilting of the lattice along that direction. This tilting, probably stabilized by the existence of misfit dislocations, helps relieve that large mismatch along that particular direction. STEM cross-sectional images of the modified layer show the homogeneity of the modified layer, revealing a constant thickness of $10 \mathrm{~nm}$ and its relatively good crystallinity.

Density functional theory (DFT) calculations confirm the high stability of the interface for a particular structural configuration, with an energy estimated to be $\gamma_{\text {int }}=3.2 \mathrm{~J} \mathrm{~m}^{-2}$. The good structural and chemical matchings justify this low value. Also, the calculations suggest that a contraction of the $\mathrm{TiO}$ bonds at the interface (the out-of-plane $\mathrm{TiO}$ distance slightly increases with the interface distance) is due to the charge transfer from $\mathrm{TiO}$ to $\mathrm{TiO}_{2}$, resulting in an interface with metallic character. 

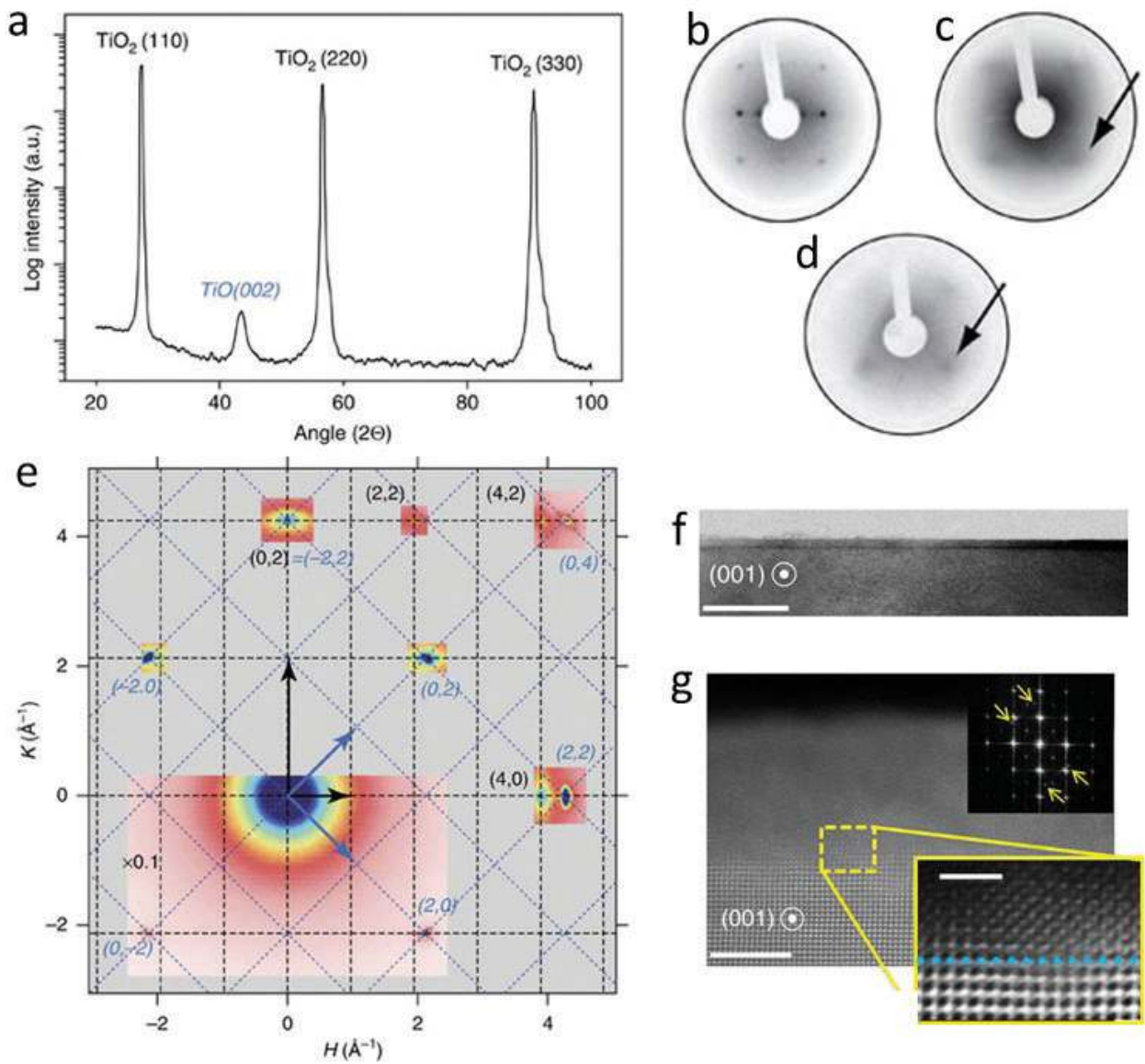

Figure 6. (a) $\theta-2 \theta$ scan of a $\mathrm{TiO}_{2}(110)$ single crystal after ion bombardment. A new reflection from $\mathrm{TiO}(002)$, not present before, emerges. (b) LEED image of the $\mathrm{TiO}_{2}(110)$ surface before LEIB, (c) during LEIB, and (d) after LEIB and a soft thermal annealing. New spots (marked with arrows), with a different symmetry, appear. They become sharper after the annealing. All diffractograms are taken at $E=63 \mathrm{eV}$. (e) X-ray diffraction HK map in reciprocal space of the ionbombarded $\mathrm{TiO}_{2}(110)$ surface, where both sets of reflections are seen: black for $\mathrm{TiO}_{2}(110)$ and blue for $\mathrm{TiO}(001)$. The two surface lattices, with their corresponding lattice vectors, are marked. (f) Scanning transmission electron microscopy (STEM) image of the irradiated layer. The transformed surface is seen. (g) Higher magnification STEM image and fast Fourier transform (top right) of the image. The yellow rectangle is a detail of the interface. Adapted with permission from Ref. [38].

All results together indicate that the initial stages of ion bombardment induce the formation of a chemically reduced and defective version of the dioxide at the initial stages, which transforms into a very disordered (or even amorphous) phase for intermediate doses, as the rutile structure is not stable for a high density of defects. The absence of a LEED pattern after medium doses supports the existence of this disordered intermediate phase. At sufficiently high doses, while preferential oxygen sputtering continues operative, the $\mathrm{TiO}(001)$ phase emerges, favored by the good registry between both oxides at the interface. 
An interesting fact is that the thickness of the modified layer $(10 \mathrm{~nm})$ is larger than the average depth where most of the ion damage is generated (around $4 \mathrm{~nm}$, according to SRIM simulations). This implies that diffusion is active, probably assisted by radiation, and also enhanced by a locally higher temperature due to the dissipation of the energetic ions. Most probably, mass transport is governed by interstitial Ti cations [41, 42] generated by ion bombardment, which migrate to the interface transforming it locally into TiO. The interface thus advances toward the bulk. When the diffusion length of the species is not sufficiently long to compensate for the receding surface (atoms are being continuously sputtered by the ion beam), then a steady state is reached, and the thickness of the modified layer saturates.

\section{Acknowledgements}

I would like to thank all my former and present collaborators, especially those participating in the works reviewed in the last section of this chapter. Financial support from Projects MAT2012-38045-C04-03 and FIS2014-61839-EXP from the Spanish MINECO is also acknowledged.

\section{Author details}

Oscar Rodríguez de la Fuente

Address all correspondence to: oscar.rodriguez@fis.ucm.es

Materials Physics Department, Universidad Complutense de Madrid, Madrid, Spain

\section{References}

[1] Grove WR. On some anomalous cases of electrical decomposition. Philos. Mag. 1853;5:203.

[2] Goldstein E. Verh. Dtsch. Phys. Ges. 1902;4:228.

[3] Sigmund P. Sputtering by ion bombardment: theoretical concepts. In: Behrisch R, editor. Sputtering by Particle Bombardment I. Springer-Verlag. Berlin Heidelberg. 1981. pp. 9-71.

[4] Gnaser H. Low-Energy Ion Irradiation of Solid Surfaces. Springer-Verlag. Berlin Heidelberg. 1999. DOI: 10.1007/BFb0110693

[5] Betz G, Wehner GK. Sputtering in multicomponent materials. In: Behrisch R, editor. Sputtering by Particle Bombardment II. Springer-Verlag. Berlin Heidelberg. 1983. pp. 11-90.

[6] van der Heide P. Secondary Ion Mass Spectrometry: An Introduction to Principles and Practices. Wiley. Hoboken, New Jersey. 2014. DOI: 10.1002/9781118916780. 
[7] Prieto P, de la Figuera J, Martín-García L, Prieto JE, Marco JF. Fourfold in-plane magnetic anisotropy of magnetite thin films grown on TiN buffered $\mathrm{Si}(001)$ by ion-assisted sputtering. J. Mater. Chem. C. 2016;4:7632. DOI: 10.1039/c6tc02152b.

[8] Taylor HS. R. Soc. London Ser. A. A theory of the catalytic surface 1925;108:105.

[9] Palacio I, Rojo JM, Rodríguez de la Fuente O. Surface defects activating new reaction paths: Formation of formate during methanol oxidation on $\mathrm{Ru}(0001)$. ChemPhysChem. 2012;13:2354. DOI: 10.1002/cphc.201200190.

[10] de la Fuente, OR, González MA, Rojo JM. Experimental evidence of two-dimensional dislocations in a reconstructed layer. Surf. Sci. 2000;454:16.

[11] de la Fuente OR, González MA, Rojo JM. Ion bombardment of reconstructed metal surfaces: From two-dimensional dislocation dipoles to vacancy pits. Phys. Rev. B. $2001 ; 6: 85420$.

[12] Carrasco E, de la Fuente OR, González MA, Rojo J. Characterising and controlling surface defects. Eur. Phys. J. B. 2004;40:421. DOI: 10.1140/epjb/e2004-00231-9.

[13] Navarro V, Rodríguez de la Fuente O, Mascaraque A, Rojo JM. Plastic properties of gold surfaces nanopatterned by ion beam sputtering. J. Phys. Cond. Matter. 2009;21:224023. DOI: 10.1088/0953-8984/21/22/224023.

[14] Rodríguez de la Fuente O, Gonzalez-Barrio MA, Navarro V, Pabon BM, Palacio I, Mascaraque A. Surface defects and their influence on surface properties. J. Phys. Cond. Matter. 2013;25:484008. DOI: 10.1088/0953-8984/25/48/484008.

[15] Cai A, Piercy P. Morphology of the rutile (110) surface after low sputter dose and annealing. Phys. Rev. B. 2002;66:115414. DOI: 10.1103/PhysRevB.66.115414

[16] Navez M, Sella C, Chaperot D. C.R. Acad. Sci. Microscopie electronique-etude de lattaque du verre par bombardement ionique. 1962;254:240.

[17] Bradley RM, Harper JME. J. Vac. Sci. Technol. A Theory of ripple topography induced by ion bombardment 1988;6:2390.

[18] Sigmund P. Phys. Rev. Theory of sputtering I. Sputtering yield of amorphous and polycrystalline targets 1964;184:383.

[19] Norton DP. Synthesis and properties of epitaxial electronic oxide thin-film materials. Mater. Sci. Eng. R. 2004;43:139. DOI: 10.1016/j.mser.2003.12.002.

[20] Keller A, Facsko S, Möller W. The morphology of amorphous $\mathrm{SiO}_{2}$ surfaces during low energy ion sputtering. J. Phys.: Condens. Matter. 2009;21:495305. DOI: 10.1088/0953-8984/21/495305.

[21] Romero-Gomez P, Palmero A, Ben T, Lozano JG, Molina SI, González-Elipe R. Surface nanostructuring of $\mathrm{TiO}_{2}$ thin films by high energy ion irradiation. Phys. Rev. B. 2010;82:115420. DOI: 10.1103/PhysRevB.82.115420. 
[22] Berkó A, Biró T, Kecskés T, Solymosi F. Self-organization of oxide nanodots generated by low energy $\mathrm{Ar}^{+}$bombardment on $\mathrm{TiO}_{2}(110)-(1 \times 2)$. Vacuum .2001;61:317.

[23] Luttrell $\mathrm{T}$, Batzill M. Nanoripple formation on $\mathrm{TiO}_{2}(110)$ by low-energy grazing incidence ion sputtering. Phys. Rev. B. 2010;82:035408. DOI: 10.1103/PhysRevB.82.035408.

[24] Kolmer M, Zebari AA, Goryl M, Buatier de Mongeot F, Zasada F, Piskorz F, Pietrzyk P, Sojka Z, Krok F, Szymonski M. Temperature-dependent orientation of self-organized nanopatterns on ion-irradiated $\mathrm{TiO}_{2}$ (110). Phys. Rev. B. 2013;88:195427. DOI: 10.1103/ PhysRevB.88.195427.

[25] Luttrell T, Li WK, Gong XQ, Batzill M. New directions for atomic steps: step alignment by grazing incident ion beams on $\mathrm{TiO}_{2}(110)$. Phys. Rev. Lett. 2009;102:166103. DOI: 10.1103/PhysRevLett.102.166103.

[26] Lee SA, Jeong H, Woo S, Hwang JY, Choi SY, Kim SD, Choi M, Roh S, Yu H, Hwang J, Kim SW, Choi WS. Phase transitions via selective elemental vacancy engineering in complex oxide thin films. Sci. Rep.. 2016;6:23649. DOI: 10.1038/srep23649.

[27] Wang Q, Zhang W, Zhang W, Zeng H. In-situ monitor of insulator to metal transition in $\mathrm{SrTiO}_{3}$ by Ar${ }^{+}$irradiation. Appl. Surf. Sci. 2016;365:84.

[28] Reagor DW, Butko VY. Highly conductive nanolayers on strontium titanate produced by preferential ion-beam etching. Nat. Mater. 2005;4:593. DOI: 10.1038/nmat1402

[29] Cooper CM, Nayar PS, Hale EB, Gerson R. Conducting strontium titanate layers produced by boron-ion implantation. J. Appl. Phys. 1979;50:2826.

[30] Singh A, Hänisch J, Matias V, Ronning F, Mara N, Pohl D, Rellinghaus B, Reagor D. Transforming insulating rutile single crystal into a fully ordered nanometer-thick transparent semiconductor. Nanotechnology 2010;21:415303. DOI: 10.1088/0957-4484/21/41/415303.

[31] Kan D, Terashima T, Kanda R, Masuno A, Tanaka K, Chu S, Kan H, Ishizumi A, Kanemitsu Y, Shimakawa Y, Takano M. Blue-light emission at room temperature from Ar-irradiated $\mathrm{SrTiO}_{3}$. Nat. Mater.. 2005;4:816. DOI: 10.1038/nmat1498.

[32] Majumder S, Paramanik D, Solanki V, Bag BP, Varma S. Bandgap tailoring of rutile $\mathrm{TiO}_{2}(110)$ via surface patterning with electron cyclotron resonance sputtering. Appl. Phys. Lett. 2011;98:053105. DOI: 10.10631/1.3549768.

[33] Rogala M, Klusek Z, Rodenbücher C, Waser R, Szot K. Quasi-two-dimensional conducting layer on $\mathrm{TiO}_{2}(110)$ introduced by sputtering as a template for resistive switching. Appl. Phys. Lett. 2013;102:131604.

[34] Gross $\mathrm{H}$, Seongshik O. Efficient resistive memory effect on $\mathrm{SrTiO}_{3}$ by ionic-bombardment. Appl. Phys. Lett. 2011;99:092105. DOI: 10.1063/1.3633114.

[35] Zheng C, Lan TC, Shueh C, Desautets RD, van Lierop J, Lin KW, Pong WT. Effect of ion bombardment on microstructural and magnetic properties of $\mathrm{Ni}_{80} \mathrm{Fe}_{20} / \alpha-\mathrm{Fe}_{2} \mathrm{O}_{3}$ thin films. Jap. J. Appl. Phys. 2014;53:06JB03. DOI: 10.7567/JJAP.53.06JB03 
[36] Aurino PP, Kalabukhov A, Tuzla N, Klein A, Erhart P, Boikov YA, Serenkov IT, Sakharov VI, Claeson T, Winkler D. Reversible metal-insulator transition of Ar-irradiated $\mathrm{LaAlO}_{3} /$ $\mathrm{SrTiO}_{3}$ interfaces. Phys. Rev. B. 2015;92:155130. DOI: 10.1103/PhysRevB.92155130.

[37] Bruno FY, Tornos J, Gutierrez del Olmo M, Sánchez-Santolino G, Nemes NM, GarciaHernandez M, Mendez B, Piqueras J, Antorrena G, Morellón L, de teresa JM, Clement M, Iborra E, León C, Santamaría J. Anisotropic magnetotransport in $\mathrm{SrTiO}_{3}$ surface electron gases generated by Ar+ irradiation. Phys. Rev. B. 2011;83:245120. DOI: 10.1103/ PhysRevB.83.245120.

[38] Pabón BM, Beltrán JI, Sánchez-Santolino G, Palacio I, López-Sánchez J, Rubio-Zuazo J, Rojo JM, Ferrer P, Mascaraque A, Muñoz MC, Varela M, Castro GR, Rodríguez de la Fuente O. Formation of titanium monoxide (001) single-crystalline thin film induced by ion bombardment of titanium dioxide (110). Nat. Comm. 2014;6:6147. DOI: 10.1038/ ncomms7147

[39] Diebold U. The surface science of titanium dioxide. Surf. Sci. Rep. 2003;48:53.

[40] Fujishima A, Zhang X, Tryk DA. $\mathrm{TiO}_{2}$ photocatalysis and related surface phenomena. Surf. Sci. Rep. 2008;63:515. DOI: 10.1016/j.surfrep.2008.10.001.

[41] Henderson MA. A surface perspective on self-diffusion in rutile $\mathrm{TiO}_{2}$. Surf. Sci. 1999;419:174.

[42] Jug K, Nair NN, Bredow T. Molecular dynamics investigation of oxygen vacancy diffusion in rutile. Phys. Chem. Chem. Phys. 2005;7:2616. 
